\title{
Finger Knuckle Identification using Principal Component Analysis and Nearest Mean Classifier
}

\author{
Shubhangi Neware \\ Research Scholar \\ Dr.C.V. Raman University \\ Bilaspur (C.G.)
}

\author{
Kamal Mehta, PhD. \\ Professor (CSE) \& Dean \\ Sagar Institute of Research and \\ Technology, Bhopal (M.P.)
}

\author{
A.S. Zadgaonkar, PhD. \\ Vice Chancellor \\ Dr.C.V. Raman University \\ Bilaspur (C.G.)
}

\begin{abstract}
The texture pattern produced by the finger knuckle bending is highly unique and makes the surface a distinctive biometric identifier. This paper presents literature survey and classification method for an emerging biometric identifier, namely Finger-Knuckle-Print (FKP), for personal identification. The FKP feature extraction is done using Principal Component Analysis (PCA) technique. Also Knuckle classification using nearest mean classifier is proposed in this paper. The experimental results from the proposed approach are promising and confirm the usefulness of this approach for personal identification.
\end{abstract}

\section{General Terms}

Principal Component Analysis (PCA), Region of Interest (ROI).

\section{Keywords}

Finger Knuckle Print (FKP), Nearest Mean Classifier, Eigen vectors, Eigen value, Eigen knuckle, and knuckle space.

\section{INTRODUCTION}

The finger-back surface, also known as dorsum of hand, can be highly useful in user identification and has not yet attracted the attention of researchers. The contact free imaging of the finger back surface is highly convenient to users. Such images can be acquired online and used to extract scale, translation and rotational invariant knuckle features for user identification [1].It is reported that the skin pattern on the finger-knuckle is highly rich in texture due to skin folds and creases, and hence, can be considered as a biometric identifier. Further, advantages of using Finger Knuckle Print (FKP) include rich in texture features, easily accessible, contact-less image acquisition, invariant to emotions and other behavioral aspects such as tiredness, stable features and acceptability in the society. Despite of these characteristics and advantages of using FKP as biometric identifier, limited work has been reported in the literature [2]. The usage of finger knuckle for personal identification has shown promising results and generated lot of interest in biometrics. However, the research efforts to investigate the utility of finger knuckle patterns for personal identification have been very limited. As a result; there is no known use of knuckle pattern in commercial or civilian applications [14]. The user acceptance for employing finger knuckle in human identification is expected to be very high as there is no stigma of personal information (such as life-line, heart-line, head-line associated with palm surface) associated with finger knuckle lines/creases [17].Proposed work is focusing on the use of appearance based method for extracting knuckle features from the finger back surface and using it for personal identification.

\section{LITERATURE REVIEW}

The finger surface posses unique patterns that have been utilized in the personal identification. Woodard and Flynn (2005) [3] have examined the fine features of finger surface for its use in the biometric system. Authors have presented promising results by using curvature and a shape-based index from finger surface features extracted from finger images. For hand data collection the Minolta 900/910 sensor was used by author. However, the work detailed in [3] does not exploit the texture information that can be simultaneously extracted from the intensity images of hands. Ribaric and Fratric (2005) [4] employed appearance based features from the finger and palm surface images for personal identification. However, the authors in [4] have employed a scanner for imaging which is very slow and, hence, not suitable for online user authentication. S. Malassiotis (2006) combines finger geometry features and color information to authenticate user hands in the cluttered background. The finger shape information is generally believed to be less discriminative and only suitable for small-scale user identification [1]. Michael K.O. Goh and Connie Tee (2009) [5] employed a bimodal palm and knuckle print recognition system using inner surface of palm and finger knuckle. Authors presented a palm print and knuckle print tracking approach to automatically detect and capture these features from low resolution video stream. No constraint is imposed and the subject can place his/her hand naturally on top of the input sensor without touching any device. The palm print and knuckle print features are extracted using Wavelet Gabor Competitive Code and Ridget Transform methods. Several decision-level fusion rules are used to consolidate the scores output by the palm print and knuckle print. The work, detailed in [4] and [5] is promising but it relies on crease and wrinkle details on the palm side (inner surface) of the fingers which are quite limited. Ajay Kumar and Ravikanth (2009) [1] [18] investigates a new approach for personal authentication using finger back surface imaging. Author uses texture pattern produced by the finger knuckle bending for identification as it is highly unique and makes the surface a distinctive biometric identifier. Finger geometry features are also extracted from the same image at the same time and integrated to further improve the useridentification accuracy of such a system. Ajay Kumar, Yingbo Zhou (2009) [14] [17] investigate a new approach for efficient and effective personal identification using Knuckle Codes. The enhanced knuckle images are employed to generate Knuckle Codes using Localized Radon Transform that can efficiently characterize random curved lines and creases. The similarity between two Knuckle Codes is computed from the minimum matching distance that can account for the variations resulting from translation and positioning of fingers. Lin Zhang, Lei Zhang and David Zhang (2009) [6] 
[16] [19] constructed data acquisition device to capture the Finger Knuckle Print images, and then an efficient FKP recognition algorithm is presented to process the acquired data .The local convex direction map of the FKP image is extracted based on which a coordinate system is defined to align the images and a Region of Interest (ROI) is cropped for feature extraction. A competitive coding scheme, which uses 2D Gabor filters to extract the image local orientation information, is employed to extract and represent the FKP features. To match two FKPs, they present a Band-Limited Phase-Only Correlation (BLPOC) based method to register the images and further to evaluate their similarity. An FKP database was established to examine the performance of the proposed system. Rui Zhao (2009) [7] presents an approach which use single knuckle-print image only to implement personal identification. Unlike most previous work, there is no need to collect a large amount of images to train the classifier. Michal Choras and R.Kazil (2010) [15] evaluated texturebased knuckle features using IIT Delhi knuckle image database. G S Badrinath, Aditya Nigam and Phalguni Gupta (2011) [2] presented an Efficient Finger-knuckle-print based Recognition System Fusing SIFT (Scale Invariant Feature Transform) and SURF (Speeded up Robust Features) Matching Scores. Corresponding features of the enrolled and the query FKPs are matched using nearest-neighbour-ratio method and then the derived SIFT and SURF matching scores are fused using weighted sum rule. Lin Zhang, Lei Zhang, David Zhang, Hailong Zhu (2011) [8] proposed Ensemble of local and global information for finger-knuckle-print recognition. Shoichiro Aoyama, Koichi Ito and Takafumi Aoki (2011) [9] proposed Finger-Knuckle-Print (FKP) recognition algorithm using Band-Limited Phase-Only Correlation (BLPOC)-based local block matching. The phase information obtained from 2D Discrete Fourier Transform (DFT) of images contains important information of image representation. The phase-based image matching, especially BLPOC-based image matching is successfully applied to image recognition tasks for biometric authentication applications. To calculate the matching score, the proposed algorithm corrects the global and local distortion between FKP images using the BLPOC-based local block matching. Zhao Rui, Lv Tao, Hou Shunyan, Shi Jianying (2011) [10] proposed a novel approach of Personal Identification based on the Fusion of Multifinger knuckle prints. Author's research work involves extracting and matching the knuckle print's line feature, fusing of the matching scores of the multi-finger knuckle prints to get the total matching score and then certifying the identity of the user.

\section{PROPOSED METHODOLOGY}

Principal component analysis an appearance based method is used to extract knuckle features. Nearest mean classification method is used to classify test knuckle image which is to be identified.

\subsection{Principal Component Analysis}

PCA is a useful statistical technique that has found application in fields such as face recognition and image compression. It is a common technique for finding patterns in data of high dimension. PCA algorithm extracts relevant information from a knuckle image [Principal Components] and encodes that information in a suitable data structure. In mathematical terms we find Eigen vectors and Eigen values of a covariance matrix of images. Where one image is just a single point in high dimensional space $[n \times n]$, where $n \times n$ are the dimensions of image. There can be many Eigen vectors for a covariance matrix but very few of them are the principle ones. Though each Eigen vector can be used to represent different amount of variations among the knuckle images. But we are only interested in principal Eigen vectors because these can account for substantial variations among a bunch of images. They can show the most significant relationship between the data dimensions. Eigenvectors with highest Eigen values are the principle component of the image set. Using these set of Eigen vectors we can construct Eigen knuckles.

Algorithm for finding Eigen knuckles for M knuckle images having similar dimensions is explained as below.

\section{Algorithm:}

(1) Collect a bunch of sample knuckle images (say three knuckle images for each person). Dimensions of all images should be same say $\mathrm{N} \times \mathrm{N} \times \mathrm{N}$. An image can be stored in an array of $(\mathrm{N} x \times \mathrm{Ny}) \times 1$ dimension [ $\Gamma$ ] which can be considered as an image vector. Therefore training set of image vectors of size $(\mathrm{Nx} \times \mathrm{Ny}) \times \mathrm{M}$ is:

$$
\left\{\Gamma_{i} \mid i=1, \ldots \ldots \ldots, M\right\}
$$

Where $\mathrm{M}$ is the number of images.

(2) Find the average image of bunch of images.

$$
\Psi=\frac{1}{M} \sum_{i=1}^{M} \Gamma_{i}
$$

(3) Find the deviated [ img1-avg, img2-avg, ........, img.n-avg] images .

$$
\Phi_{i}=\Gamma_{i}-\Psi ; i=1 \ldots . . M
$$

(4) Calculate the covariance matrix.

$$
\mathbf{C}=\mathbf{A} \mathbf{A}^{\mathbf{T}}
$$

$$
C=\left[\begin{array}{cccc}
c(1,1) & c(1,2) & \ldots & c(1, d) \\
c(2,1) & c(2,2) & \ldots . & c(2, d) \\
: & : & & : \\
c(d, 1) & c(d, 2) & \ldots . & c(d, d)
\end{array}\right]
$$

Where $A=\left\lfloor\Phi_{1, \ldots \ldots,} \Phi_{M}\right\rfloor$

But the problem with this approach is that we may not be able to complete this operation for a bunch of images because covariance matrix will be very huge. For example covariance matrix for a knuckle image of size ( $\mathrm{Nx} x \mathrm{Ny})$ pixels is of size $(\mathrm{P} \times \mathrm{P}), \mathrm{P}$ being $(\mathrm{Nx} \times \mathrm{Ny})$. This covariance matrix is very hard to work with due to its huge dimension causing computational 
complexity. It is very hard or may be practically impossible to store that matrix. Also finding that matrix will require considerable computational requirements. So for solving this problem we first compute the matrix $\mathbf{L}$.

$$
L=A^{T} A
$$

And then find the Eigen Vectors [V] related to it

$$
\mathrm{V}_{\mathrm{x}}(\mathrm{x}=1 \ldots \ldots \ldots . . . \mathrm{M})
$$

Eigen Vectors for covariance matrix $\mathrm{C}$ can be found by

$$
U=\left[u_{1}, \ldots ., u_{M}\right]=\left[\Phi_{1, \ldots \ldots .,} \Phi_{M}\right]\left[V_{1, \ldots \ldots \ldots .,} V_{M}\right]=A . V
$$

Where

$\mathbf{U}_{\mathbf{x}}(\mathbf{x}=\mathbf{1} \ldots \ldots \ldots . . . \mathbf{M})$ are Eigen Vectors for $\mathrm{C}$.

(5) Using these Eigen vectors, we can construct Eigen knuckles. But we are interested in the Eigen vectors with high Eigen values. So Eigen vectors with less than a threshold Eigen value can be dropped .So we will keep only those images which correspond to the highest Eigen values. This set of images is called as Knuckle Space. When a new test image is to be classified, then it is projected onto the Eigen knuckle space and nearest mean algorithm is used for the classification of the test image vector in the standard Eigen knuckle method; that is, the test image is assumed to belong to the nearest class by calculating the Euclidean distance of the test image vector to the mean of each class of the training image vectors [21].

\subsection{Algorithm to classify the test knuckle image}

Step 1: Input the image and calculate test image vector

$$
\Gamma_{\mathrm{T}}
$$

Step 2: Compute mean subtracted image for test image

$$
\Phi_{\mathrm{T}}=\Gamma_{\mathrm{T}}-\Psi
$$

is the difference of the test image from the mean image.

Step 3: Projection of test image on "Knuckle Space"

$$
\omega_{k}=v_{k}^{\mathrm{T}} \cdot \Phi_{\mathrm{T}}=v_{k}^{\mathrm{T}} \cdot\left(\Gamma_{\mathrm{T}}-\Psi\right)
$$

is the projection of a test knuckle image on each of the eigenvector where

$\mathrm{k}=1,2, \ldots, \mathrm{M}^{\prime}$

\section{Step 4: Compute the weight matrix for test image}

Weight matrix describes the contribution of each Eigen knuckle in representing the input knuckle image, treating the Eigen knuckle as a basis set for knuckle images.<smiles>[3H]C#CC=[18O]</smiles>

is the representation of the test image in the Eigen knuckle space and its size is $\left(\mathrm{M}^{\prime} \mathrm{x} 1\right)$.

Step 5: Compute the average weight matrix for each class (Average class projection into "knuckle space")

Image database contains more than one knuckle image for same finger per individual in order to increase the robustness to minor changes in illumination and slight variations of view angles. A class of images for an individual can be formed and this class can be considered as the representative image vector of that class. For an individual having qi images in the database, the average of the projections of each class is the mean of all the projected image vectors in that class. In mathematical terms;

\section{Average Class Projection is}

$$
\Omega_{\Psi}=\frac{1}{q_{i}} \sum_{i=1}^{q_{i}} \Omega_{i}
$$

This average class projection can be used as one of the vectors (representing an image class instead of an image vector) to compare with the test image vector. The test image is assumed to belong to the nearest class by calculating the Euclidean distance of the test image vector to the mean of each class of the training image vectors.

\subsection{Comparing Test image with class image}

Nearest mean classifier technique is used here to find a class nearest to the test image. In the nearest mean classifier, the Euclidean distance from each class mean (in this case) is computed for the decision of the class of the test data.A similarity measure is defined as the distance between the test image vector and $\mathrm{i}$-th knuckle class.

$$
\delta_{i}=\left\|\Omega_{\mathrm{T}}-\Omega_{\Psi i}\right\|=\sqrt{\sum_{k=1}^{M_{t}}\left(\Omega_{\mathrm{T} k}-\Omega_{\Psi i k}\right)^{2}}
$$

is the Euclidean distance between projections for all classes.

\section{TESTING AND EXPERIMENTAL RESULTS}

Performance of the proposed system is tested on publicly available "IIT Delhi finger knuckle database version 1.0" [22]. Sample knuckle images are as shown in figure 1. Three knuckle image samples of same person are assigned to one class. Figure 2 shows test knuckle image which is to be 
identified .Test knuckle image is then classified using nearest mean classifier. The result is produced in terms of nearest class number with the smallest distance from the mean of that class as shown in Figure 3. The mean knuckle image is as shown in Figure 4. Figure 5 shows the projection of Test image on the knuckle space.
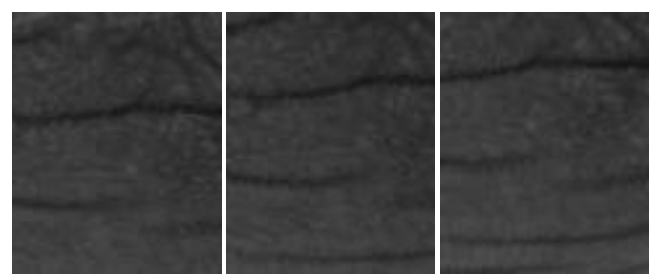

Class 4
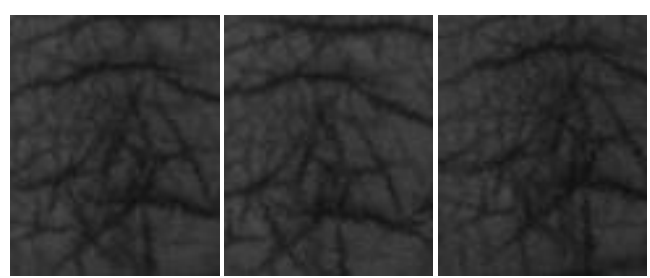

Class3
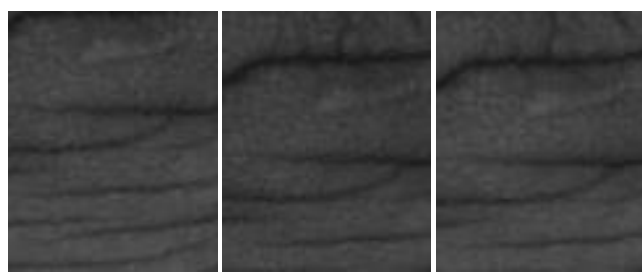

Class 2
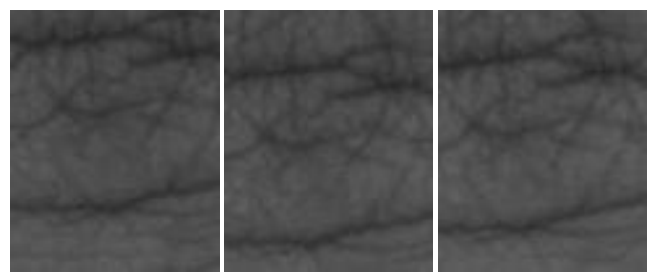

Class 1

Figure 1. Sample knuckle images from database

The proposed system was entirely implemented in Matlab 7, using Image Processing Toolbox.

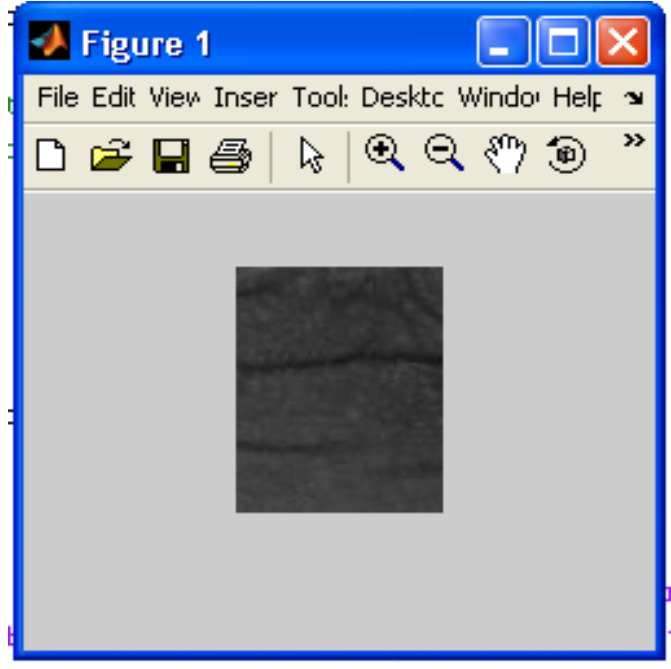

Figure 2. Test knuckle image

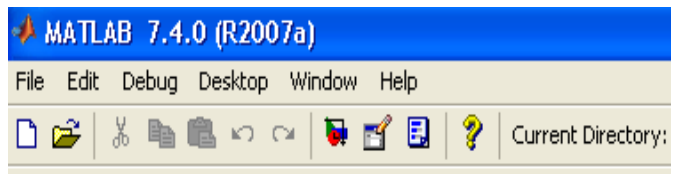

Shortcuts [] How to Add ] What's New

(i) To get started, select MATLAB Help or Demos from the Help menu.

The nearest class is number

4

with a distance equal to

239.9062

The distance from Knuckle space is 551.2494

Figure 3. Result of nearest mean classification

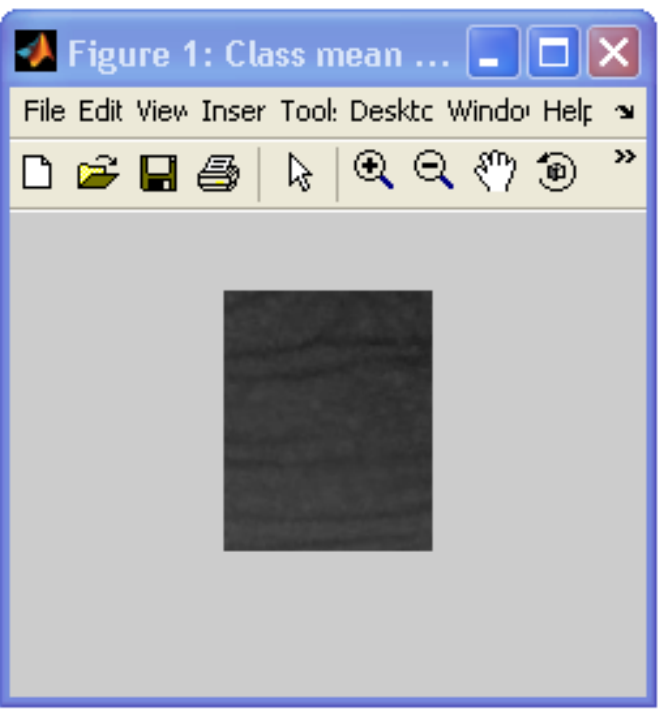

Figure 4. Mean of class 4 


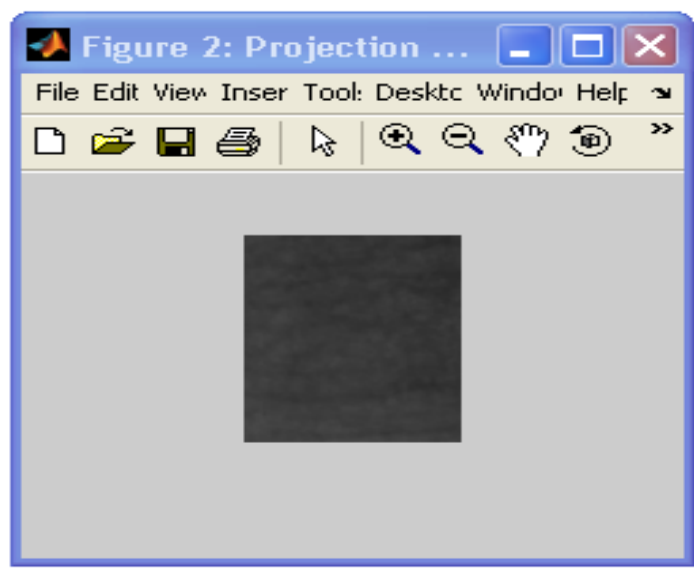

Figure 5. Projection of test image on knuckle space

Correct recognition rate (CRR) for identification is used to measure the performance of the system and is found satisfactory. CRR of the system is given as

$\mathrm{CRR}=(\mathrm{X} 1 / \mathrm{X} 2) \times 100$

Where $\mathrm{X} 1$ denotes the total number of correct recognitions of FKP images and X2 denotes the total number of persons in database. Table 1 shows performance of proposed system and other related work.

Table 1: Performance of proposed system and others

\begin{tabular}{|l|l|}
\hline Matching Technique & CRR (\%) \\
\hline Correlation Coefficient [ 3] & $91 \%$ \\
\hline Knuckle code [14] & $98.6 \%$ \\
\hline Angular distance [16] & $97 \%$ \\
\hline Proposed system & $96 \%$ \\
\hline
\end{tabular}

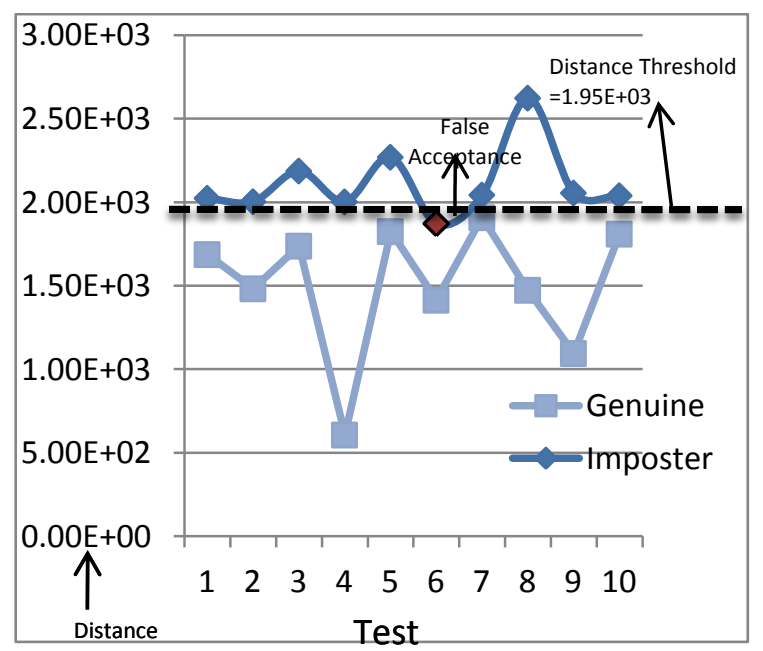

Figure 6. Distribution of imposter and genuine matching distance

At given distance threshold, the probability of accepting imposter known as false acceptance rate (FAR) and probability of rejecting genuine user is known as false rejection rate (FRR) are obtained. To decide best threshold value testing of proposed system is carried out both on imposter and genuine knuckle images as shown in figure 6.

\section{CONCLUSION}

This Paper Presented a novel approach for personal identification using finger knuckle image. Appearance based method namely Principal component analysis for feature extraction is presented in detailed. Nearest mean classification method is employed to classify knuckle images. Experimental results showing working of nearest mean classification are presented in section 4. The proposed methods for feature extraction and classification have been quite effective in achieving high performance in knuckle classification.

Proposed work consider single finger knuckle image for identification. In future this can be extended by considering multiple finger knuckle images. Finger geometry and knuckle blending information may also be combined and used for person identification. Variations in knuckle creases due to some disease may degrade the performance, this requires further investigation. In near future we plan to test this method on large database

\section{REFERENCES}

[1] Kumar A and Ravikanth C, "Personal authentication using finger knuckle surface", IEEE Transactions on Information Forensics and Security, 4(1):98 -110, 2009.

[2] Badrinath G S, Nigam A. and Gupta P, "An Efficient Finger-knuckle-print based Recognition System Fusing SIFT and SURF Matching Scores", Information and communication Security, pp374387, 2011.

[3] Woodard D.L., Flynn P.J., "Finger surface as a biometric identifier", CVIU, vol. 100, pp. 357384, 2005.

[4] Ribaric S, Fratric I., "An online biometric authentication system based on eigenfingers and finger-geometry", presented at the 13th Eur. Signal Processing Conf., Antalya, Turkey, Sep. 2005.

[5] Michael K.O, Connie T, Andrew B.J., "Bimodal Palmprint and Knuckleprint Recognition system”, Journal of IT in Asia, Vol 3,2010.

[6] Zhang L, Zhang L, Zhang D, Zhu H, "Online finger-knuckle-print verification for personal authentication",Pattern Recognition, 43(7):2560 2571, Elsevier, July 2010.

[7] Zhao R, "A Novel Approach of Personal Identification Based on Single Knuckleprint Image", Asia-Pacific Conference on Information Processing18-19 July 2009.

[8] Zhang L, Zhang L, Zhang D, Zhu H,"Ensemble of local and global information for finger-knuckleprint recognition", Pattern Recognition, 44(9):1990 - 1998, 2011. 
[9] Shoichiro A, Koichi I, Takafumi A, "Finger-KnucklePrint Recognition Using BLPOC-Based Local Block Matching”, IEEE, 2011.

[10] Rui Z, Tao L, Shunyan H, Jianying S, “ A Novel Approach of Personal Identification Based on the Fusion of Multifinger Knuckleprints", Advances in information Sciences and Service Sciences(AISS) Volume3, Number10,November 2011.

[11] Kanta Ratha N, Bolle R, "Automatic Fingerprint Recognition System”, Springer, pp 17-18.

[12] www.biometrics.gov

[13] Gupta P, Rattani A, Mehrotra H, Kaushik A, "Multimodal biometrics system for efficient human Recognition", Biometric Technique for human Identification III,Proceedings of SPIE,Apr.2006.

[14] Kumar A and Zhou Y, "Human identification using knuckle codes", Proceedings BTAS, Washington, 2009.

[15] Choras M, Kazil R, "Knuckle Biometrics Based on Texture Features", International Workshop on Emerging Techniques and Challenges for Hand-Based Biometrics (ETCHB), IEEE, 2010.

[16] Zhang L, Zhang L, Zhang D, "Finger-Knuckle Print: A New Biometric Identifier", Image Processing ICIP, IEEE International Conference, pp1981-84, Nov2009.
[17] Kumar A, Zhou Y, "Personal Identification using Finger Knuckle Orientation Features", Electronics Letters ,vol. 45, no. 20, September 2009.

[18] Kumar A, Ch Ravikanth, "Biometric Authentication Using Finger Back Surface", Computer Vision and Pattern Recognition, IEEE Conference, pp1-6, June2007.

[19] Zhang L, Zhang L, Zhang D, "Finger-KnucklePrint Verification Based on Band-Limited PhaseOnly Correlation", Proceedings of the 13th International Conference on Computer Analysis of Images and Patterns, pp. 141-148,Springer, 2009.

[20] Jain A, Kumar A, "Biometrics of Next Generation: An Overview", Second Generation Biometrics, Springer, 2010.

[21] Turk,M. and A. Pentland, Eigenfaces for Recognition. Journal of Cognitive Neuroscience, 3(1),1991,71-86.

[22]http://www4.comp.polyu.edu.hk/ CSajaykr/IITD/ii td knuckle.htm 